\title{
INITIAL BOUNDARY VALUE PROBLEMS OF THE DEGASPERIS-PROCESI EQUATION
}

\author{
JOACHIM ESCHER \\ Institute for Applied Mathematics, Leibniz University of Hannover \\ D-30167 Hannover, Germany \\ E-mail:escher@ifam.uni-hannover.de \\ ZHAOYANG YIN \\ Department of Mathematics, Zhongshan University \\ 510275 Guangzhou, China \\ E-mail: mcsyzy@mail.sysu.edu.cn \\ Institute for Applied Mathematics, Leibniz University of Hannover \\ D-30167 Hannover, Germany \\ E-mail:yin@ifam.uni-hannover.de
}

\begin{abstract}
We mainly study initial boundary value problems for the Degasperis-Procesi equation on the half line and on a compact interval. By the symmetry of the equation, we can convert these boundary value problems into Cauchy problems on the line and on the circle, respectively. Applying thus known results for the equation on the line and on the circle, we first obtain the local well-posedness of the initial boundary value problems. Then we present some blow-up and global existence results for strong solutions. Finally we investigate global and local weak solutions on the half line and on a compact interval, respectively. One interesting result is that the corresponding strong solution to the Degasperis-Procesi equation on the half line blows up in finite time provided the initial potential, assumed nonpositive, is not identically zero. Another one is that all global strong solutions to the Degasperis-Procesi equation on a compact interval blow up in finite time.
\end{abstract}

2000 Mathematics Subject Classification: 35G25, 35L05.

Key words and phrases: Degasperis-Procesi equation, initial boundary value problems, local well-posedness, blow-up and global existence, global and local weak solutions.

Research of the second author partially supported by the AvH Foundation and the NNSF of China (No. 10531040).

The paper is in final form and no version of it will be published elsewhere. 
1. Introduction. Recently, Degasperis and Procesi [17] studied the following family of third order dispersive conservation laws,

$$
u_{t}+c_{0} u_{x}+\gamma u_{x x x}-\alpha^{2} u_{t x x}=\left(c_{1} u^{2}+c_{2} u_{x}^{2}+c_{3} u u_{x x}\right)_{x},
$$

where $\alpha, c_{0}, c_{1}, c_{2}$, and $c_{3}$ are real constants and indices denote partial derivatives. In [17] the authors found that there are only three equations that satisfy the asymptotic integrability condition within this family: the KdV equation, the Camassa-Holm equation and the Degasperis-Procesi equation.

If $\alpha=c_{2}=c_{3}=0$, then Eq.(1.1) becomes the well-known KdV equation which describes the unidirectional propagation of waves at the free surface of shallow water under the influence of gravity. In this model $u(t, x)$ represents the wave's height above a flat bottom, $x$ is proportional to distance in the direction of propagation and $t$ is proportional to the elapsed time. The $\mathrm{KdV}$ equation is completely integrable and its solitary waves are solitons [37]. The Cauchy problem of the KdV equation has been studied extensively, see $[29,40]$ and the citations therein. It is shown that the KdV equation is globally well-posed for $u_{0} \in L^{2}(R)$, cf. [40]. It is observed that the $\mathrm{KdV}$ equation does not accomodate wave breaking (by wave breaking we understand that the wave remains bounded but its slope becomes unbounded in finite time [42]).

For $c_{1}=-\frac{3}{2} c_{3} / \alpha^{2}$ and $c_{2}=c_{3} / 2$, Eq.(1.1) becomes the Camassa-Holm equation, modeling the unidirectional propagation of shallow water waves over a flat bottom, $u(t, x)$ stands for the fluid velocity at time $t \geq 0$ in the spatial $x \in R$ direction (see [3, 18, 27]). The Camassa-Holm equation has a bi-Hamiltonian structure [23] and is completely integrable (see [1]). Its solitary waves are peaked, cf. $[3,4]$. They are orbitally stable (see [15]).

Initial value problems of the Camassa-Holm equation on the line and on the circle have been studied extensively. It has been shown that this equation is locally well-posed $[9,10,39]$ for initial data $u_{0} \in H^{s}(I)$ with $s>\frac{3}{2}$, where $I=R$ or $I=S=R / Z$. More interestingly, it has global strong solutions $[7,9,10,34]$ and also finite time blow-up solutions $[7,9,10,11,13,34]$. On the other hand, it has global weak solutions in $H^{1}(I)$, cf. $[2,14,34,43]$. The advantage of the Camassa-Holm equation in comparison with the $\mathrm{KdV}$ equation lies in the fact that the Camassa-Holm equation has peaked solitons and models wave breaking [4].

If $c_{1}=-2 c_{3} / \alpha^{2}$ and $c_{2}=c_{3}$ in Eq.(1.1), then, after rescaling, shifting the dependent variable, and applying a Galilean boost [16], we find the Degasperis-Procesi equation of the form

$$
u_{t}-u_{t x x}+4 u u_{x}=3 u_{x} u_{x x}+u u_{x x x}, \quad t>0, x \in R .
$$

Degasperis, Holm and Hone [16] proved the formal integrability of Eq.(1.2) by constructing a Lax pair. They also showed that Eq.(1.2) has a bi-Hamiltonian structure with an infinite sequence of conserved quantities and that it admits exact peakon solutions which are analogous to the Camassa-Holm peakons.

The Degasperis-Procesi equation can be regarded as a model for nonlinear shallow water dynamics and its asymptotic accuracy is the same as for the Camassa-Holm shallow water equation [19]. In this model, $u(t, x)$ stands for the fluid velocity at time $t$ in the 
spatial $x$ direction. Dullin, Gottwald and Holm [19] showed that the Degasperis-Procesi equation can be obtained from the shallow water elevation equation by an appropriate Kodama transformation. Lundmark and Szmigielski [33] presented an inverse scattering approach for computing $n$-peakon solutions to Eq.(1.2). Holm and Staley [26] studied stability of solitons and peakons numerically.

After the Degasperis-Procesi equation (1.2) was derived, many papers were devoted to its study, cf. $[5,25,30,31,32,36,44,45,46,47]$ and the citations therein. For example, Yin proved local well-posedness of Eq.(1.2) with initial data $u_{0} \in H^{s}(R), s>\frac{3}{2}$ on the line [44] and on the circle [45]. In these two papers the precise blow-up scenario and a blow-up result were derived. The global existence of strong solutions and global weak solutions to Eq.(1.2) are also investigated in [46, 47]. Recently, Lenells [30] classified all weak traveling wave solutions. Matsuno [36] studied multisoliton solutions and their peakon limits. Analogous to the case of Camassa-Holm equation [8], Henry [25] showed that smooth solutions to Eq.(1.2) have infinite speed of propagation. Coclite and Karlsen [5] also obtained global existence results for entropy solutions in $L^{1}(R) \cap B V(R)$ and in $L^{2}(R) \cap L^{4}(R)$

Despite the similarities to the Camassa-Holm equation, it should be emphasized that these two equations are truly different. One of the important features of Eq.(1.2) is that it has not only peakon solitons [16, 46], but also shock waves [6, 32, 21].

On the other hand, the isospectral problem for Eq.(1.2) has the third-order equation in the Lax pair

$$
\psi_{x}-\psi_{x x x}-\lambda y \psi=0,
$$

cf. [16], while the isospectral problem for the Camassa-Holm equation is the second order equation

$$
\psi_{x x}-\frac{1}{4} \psi-\lambda y \psi=0,
$$

cf. [3] (in both cases $y=u-u_{x x}$ ).

Another indication of the fact that there is no simple transformation of Eq.(1.2) into the Camassa-Holm equation is the entirely different form of conservation laws for those two equations $[3,16]$. Furthermore, the Camassa-Holm equation is a re-expression of geodesic flow on the diffeomorphism group [12] or on the Bott-Virasoro group [38]. Up to now, no geometric derivation of the Degasperis-Procesi equation is available.

It turns out in [31] that the conservation laws of the Degasperis-Procesi equation are much weaker than those of the Camassa-Holm equation. Although the bi-Hamiltonian structure of Eq.(1.2) provides an infinite number of conservation laws [16], the conservation laws of the Degasperis-Procesi equation cannot guarantee the boundedness of the slope of wave, and there is no way to find conservation laws controlling the $H^{1}$-norm, which plays important role in studying the Camassa-Holm equation.

Recently, several new global existence and blow-up results for strong solutions to the Degasperis-Procesi equation on the line were presented in [31]. Global weak solutions and the blow-up structure for this equation on the line were also investigated in [20]. It [31] is shown that the first blow-up must occur as wave breaking and shock waves possibly appear afterwards. 
Initial boundary value problems for the Camassa-Holm on the half line and the compact interval $[0,1]$ have been recently studied in $[28,35,22]$. However, initial boundary value problems for the Degasperis-Procesi equation on the half line and on the compact interval $[0,1]$ seem not yet to have been investigated. In this paper, we mainly investigate initial value problems for the Degasperis-Procesi equation on the half line and on the compact interval $[0,1]$ with Dirichlet boundary condition, which corresponds to nonslip boundary condition, this means that the boundary of $I$ is solid and at rest. The aim of this paper is to develop a new approach to study two kinds of initial boundary value problems for the Degasperis-Procesi equation on the half line with the initial data $u_{0} \in H^{s}\left(R_{+}\right) \cap H_{0}^{1}\left(R_{+}\right), s>\frac{3}{2}$ and on the compact interval with the initial data $u_{0} \in H^{s}([0,1]) \cap H_{0}^{1}([0,1]), s>\frac{3}{2}$. By this new approach, we can convert initial boundary value problems of the Degasperis-Procesi equation on the half line and on the compact interval into Cauchy problems of the Degasperis-Procesi equation on the whole line and of the periodic Degasperis-Procesi equation. Applying the known results for the DegasperisProcesi equation on the whole line and the periodic Degasperis-Procesi equation, we first obtain local well-posedness results, then present blow-up and global existence results for strong solutions, finally obtain global weak solutions for the equation on the half line and local weak solutions on the compact interval.

Our paper is organized as follows. In Section 2, we first present a new approach. In Section 3, by this new approach, we can investigate initial boundary value problems of the Degasperis-Procesi equation on the half line. One interesting result is that the corresponding strong solutions to the Degasperis-Procesi equation on the half line blows up in finite time provided the initial potential $y_{0}(x)=u_{0}-u_{0, x x} \not \equiv 0$ and $y_{0}(x) \leq 0$ for all $x \in R_{+}$. In Section 4, we study initial boundary value problems of the Degasperis-Procesi equation on the compact interval. An interesting result of our analysis shows that the Degasperis-Procesi equation on a compact interval possesses no nontrivial global strong solutions.

Notation. In the following, we denote by $*$ the spatial convolution. Given a Banach space $X$, we denote its norm by $\|\cdot\|_{X}$. The Banach space of all bounded linear operators mapping $X$ into another $Y$ is denoted by $\mathcal{L}(X, Y)$. If $I$ is any interval of $R$ we write $M(I)$ for all Radon measures on $I$ and $M^{+}(I)$ for all positive Radon measures on $I$.

2. Preliminaries. In this section, we will present several useful lemmas which are used in the sequel.

Let $I \in R$ be an interval and let $[\cdot, \cdot]_{\theta}, \theta \in(0,1)$, denote the complex interpolation method. Then it follows from Theorem 2.10.1 and formula 2.4.2/11 in [41]

$$
H^{\theta}(I)=\left[L^{2}(I), H^{1}(I)\right]_{\theta} .
$$

Definition 2.1. Let $E, F$ be Banach spaces. An operator $r \in \mathcal{L}(E, F)$ is called a retraction from $E$ onto $F$ if there exists a $r^{c} \in \mathcal{L}(F, E)$ such that $r \circ r^{c}=i d_{F}$. In this case $r^{c}$ is called a co-retraction for $r$.

Then we have the following lemmas. 
Lemma 2.1. Assume that $E_{1} \subset E_{0}$ and $F_{1} \subset F_{0}$ and that $r$ is a retraction from $E_{j}$ onto $F_{j}, j=0,1$. Moreover, assume that the restriction of the co-retraction $r^{c} \in \mathcal{L}\left(F_{0}, E_{0}\right)$ to $F_{1}$ is bounded in $E_{1}$. Let further

$$
E_{\theta}:=\left[E_{0}, E_{1}\right]_{\theta}, \quad F_{\theta}:=\left[F_{0}, F_{1}\right]_{\theta}
$$

for some $\theta \in(0,1)$. Then $r$ is a retraction from $E_{\theta}$ onto $F_{\theta}$.

Proof. By assumption $r \in \mathcal{L}\left(E_{j}, F_{j}\right)$ and $r^{c} \in \mathcal{L}\left(F_{j}, E_{j}\right)$ with $r \circ r^{c}=i d_{F_{j}}$. Thus, interpolation yields $r \in \mathcal{L}\left(E_{\theta}, F_{\theta}\right)$ and $r^{c} \in \mathcal{L}\left(F_{\theta}, E_{\theta}\right)$ with $r \circ r^{c}=i d_{F_{\theta}}$.

Lemma 2.2. Given $v \in L^{2}\left(R_{+}\right)$and $w \in L^{2}(R)$. Let

$$
(\varepsilon v)(x)= \begin{cases}v(x), & \text { if } x \geq 0, \\ v(-x), & \text { if } x<0,\end{cases}
$$

and $(r w)(x):=w(x), x \geq 0$. Then

$$
r \in \mathcal{L}\left(L^{2}(R), L^{2}\left(R_{+}\right)\right) \cap \mathcal{L}\left(H^{1}(R), H^{1}\left(R_{+}\right)\right)
$$

is a retraction and $\varepsilon$ is a co-retraction for $r$. Moreover, given $s \in[0,1]$, we have

$$
\varepsilon \in \mathcal{L}\left(H^{s}\left(R_{+}\right), H^{s}(R)\right) .
$$

Proof. The assertion $r \in \mathcal{L}\left(L^{2}(R), L^{2}\left(R_{+}\right)\right) \cap \mathcal{L}\left(H^{1}(R), H^{1}\left(R_{+}\right)\right)$is obvious. It is also clear that $\varepsilon \in \mathcal{L}\left(L^{2}\left(R_{+}\right), L^{2}(R)\right)$ and that $r \circ \varepsilon=i d_{L^{2}\left(R_{+}\right)}$. Next we show that $\varepsilon \in$ $\mathcal{L}\left(H^{1}(R), H^{1}\left(R_{+}\right)\right)$.

Given $u \in C^{1}\left(R_{+}\right) \cap H^{1}\left(R_{+}\right)$. Then it follows that $u \in C_{l o c}^{0,1}\left(R_{+}\right)$. In view of the definition of $\varepsilon$ in Lemma 2.2 , we have that $\varepsilon u \in C_{l o c}^{0,1}(R)$. Note that locally uniformly Lipschitz continuous functions are weakly differentiable, cf. [24], that is $\varepsilon u \in W_{l o c}^{1,1}(R)$ and

$$
(\varepsilon u)^{\prime}(x)= \begin{cases}u^{\prime}(x), & \text { if } x>0 \\ 0, & \text { if } x=0 \\ -u^{\prime}(-x), & \text { if } x<0\end{cases}
$$

A direct calculation shows that

$$
\begin{aligned}
\|(\varepsilon u)(x)\|_{H^{1}(R)}^{2} & =\|(\varepsilon u)(x)\|_{L^{2}(R)}^{2}+\left\|(\varepsilon u)^{\prime}(x)\right\|_{L^{2}(R)}^{2} \\
& =2\|u(x)\|_{L^{2}\left(R_{+}\right)}^{2}+2\left\|u^{\prime}(x)\right\|_{L^{2}\left(R_{+}\right)}^{2}=2\|u(x)\|_{H^{1}\left(R_{+}\right)}^{2} .
\end{aligned}
$$

Since $C^{1}\left(R_{+}\right) \cap H^{1}\left(R_{+}\right)$is dense in $H^{1}\left(R_{+}\right)$, the above relation shows

$$
\varepsilon \in \mathcal{L}\left(H^{1}(R), H^{1}\left(R_{+}\right)\right) .
$$

This implies that $\varepsilon$ is a co-retraction for $r$. By Lemma 2.1, we obtain that for fixed $s \in[0,1], \varepsilon \in \mathcal{L}\left(H^{s}\left(R_{+}\right), H^{s}(R)\right)$.

Lemma 2.3. Given $s \in\left(\frac{3}{2}, 2\right]$ and $v \in H^{s}\left(R_{+}\right) \cap H_{0}^{1}\left(R_{+}\right)$. Let furthermore

$$
\tilde{v}(x)= \begin{cases}v(x), & \text { if } x \geq 0, \\ -v(-x), & \text { if } \quad x<0 .\end{cases}
$$

Then $\tilde{v}(x) \in H^{s}(R)$. 
Proof. From assumption $v \in H^{s}\left(R_{+}\right) \cap H_{0}^{1}\left(R_{+}\right)$, one can easily obtain

$$
\begin{aligned}
\lim _{x \rightarrow 0^{-}} \tilde{v}^{\prime}(x) & =\lim _{x \rightarrow 0^{-}} \frac{\tilde{v}(x)-\tilde{v}(0)}{x-0}=\lim _{x \rightarrow 0^{-}} \frac{-v(-x)}{x}=\lim _{x \rightarrow 0^{-}} v^{\prime}(-x)=v^{\prime}(0) \\
& =\lim _{x \rightarrow 0^{+}} v^{\prime}(x)=\lim _{x \rightarrow 0^{+}} \frac{v(x)}{x}=\lim _{x \rightarrow 0^{+}} \frac{\tilde{v}(x)-\tilde{v}(0)}{x-0}=\lim _{x \rightarrow 0^{+}} \tilde{v}^{\prime}(x) .
\end{aligned}
$$

This implies that $\tilde{v} \in H^{1}(R)$ and $(\tilde{v})^{\prime}=\varepsilon\left(v^{\prime}\right)$. Note that if $u \in H^{1}(I)$ and $\sigma \in\left(\frac{1}{2}, 1\right]$, then $u \in H^{1+\sigma}(I)$ iff $u^{\prime} \in H^{\sigma}(I)$. Let $\sigma:=s-1 \in\left(\frac{1}{2}, 1\right]$, then we have $v^{\prime} \in H^{\sigma}\left(R_{+}\right)$. By Lemma 2.2, we obtain $(\tilde{v})^{\prime}=\varepsilon\left(v^{\prime}\right) \in H^{\sigma}(R)$. This shows that $\tilde{v} \in H^{1+\sigma}(R)=H^{s}(R)$.

REMARK 2.1. For $s>2$, under the same assumption of Lemma 2.3, one cannot deduce $\tilde{v} \in H^{s}(R)$ generally.

In order to obtain $\tilde{v} \in H^{s}(R)$, one has to add additional conditions. For this we let $k \in N \backslash\{0\}$ and for $2 k+\frac{1}{2}<s \leq 2 k+2$ we set

$$
D^{s}\left(R_{+}\right)=\left\{v \in H^{s}\left(R_{+}\right) \mid v^{(2 k)}(0)=v^{(2 k-2)}(0)=\cdots=v(0)=0\right\} .
$$

Applying an induction argument and following the proof of Lemma 2.3, we can obtain the following lemma.

Lemma 2.4. Given $s \in\left(\frac{5}{2},+\infty\right)$ and $v \in D^{s}\left(R_{+}\right)$. Let furthermore

$$
\tilde{v}(x)= \begin{cases}v(x), & \text { if } \quad x \geq 0, \\ -v(-x), & \text { if } \quad x<0 .\end{cases}
$$

Then $\tilde{v}(x) \in H^{s}(R)$.

Next, one can obtain the following two lemmas for the case of $I=[0,1]$ by following the proofs of Lemmas 2.2-2.3.

Lemma 2.5. Given $v \in L^{2}([0,1])$ and $w \in L^{2}([-1,1])$. Let

$$
\left(\varepsilon_{1} v\right)(x)= \begin{cases}v(x), & \text { if } \quad x \in[0,1], \\ v(-x), & \text { if } \quad x \in[-1,0),\end{cases}
$$

and $\left(r_{1} w\right)(x):=w(x), x \in[0,1]$. Then

$$
r_{1} \in \mathcal{L}\left(L^{2}([-1,1]), L^{2}([0,1])\right) \cap \mathcal{L}\left(H^{1}([-1,1]), H^{1}([0,1])\right)
$$

is a retraction and $\varepsilon_{1}$ is a co-retraction for $r_{1}$. Moreover, given $s \in[0,1]$, we have

$$
\varepsilon_{1} \in \mathcal{L}\left(H^{s}([0,1]), H^{s}([-1,1])\right) .
$$

LEMMA 2.6. Given $s \in\left(\frac{3}{2}, 2\right]$ and $v \in H^{s}([0,1]) \cap H_{0}^{1}([0,1])$. Let furthermore

$$
\tilde{v}(x)= \begin{cases}v(x), & \text { if } x \in[0,1], \\ -v(-x), & \text { if } x \in[-1,0] .\end{cases}
$$

Then $\tilde{v}(x) \in H^{s}([-1,1]) \cap H_{0}^{1}([-1,1])$.

REMARK 2.2. For $s>2$, under the same assumptions of Lemma 2.6, one cannot deduce $\tilde{v}(x) \in H^{s}([-1,1]) \cap H_{0}^{1}([-1,1])$ generally. 
In order to obtain $\tilde{v}(x) \in H^{s}([-1,1]) \cap H_{0}^{1}([-1,1])$, one has to add additional conditions. For this we let $k \in N \backslash\{0\}$ and for $2 k+\frac{1}{2}<s \leq 2 k+2$ we set

$$
\begin{aligned}
D^{s}([0,1])=\left\{v \in H^{s}([0,1]) \mid v^{(2 k)}(0)\right. & =v^{(2 k)}(1)=v^{(2 k-2)}(0) \\
& \left.=v^{(2 k-2)}(1)=\cdots=v(0)=v(1)=0\right\} .
\end{aligned}
$$

Applying an induction argument and following the proof of Lemma 2.3, we can obtain the following lemma.

Lemma 2.7. Given $s \in\left(\frac{5}{2},+\infty\right)$ and $v \in D^{s}([0,1])$. Let furthermore

$$
\tilde{v}(x)= \begin{cases}v(x), & \text { if } \quad x \in[0,1] \\ -v(-x), & \text { if } \quad x \in[-1,0]\end{cases}
$$

Then $\tilde{v}(x)$ belongs to

$$
\begin{aligned}
D^{s}([-1,1])=\left\{v \in H^{s}([-1,1]) \mid v^{(2 k)}(-1)\right. & =v^{(2 k)}(1)=v^{(2 k-2)}(-1) \\
& \left.=v^{(2 k-2)}(1)=\cdots=v(-1)=v(1)=0\right\} .
\end{aligned}
$$

3. The case of the half line. In the section, we will investigate initial boundary value problems of the Degasperis-Procesi equation with initial data $u_{0} \in H^{s}\left(R_{+}\right) \cap H_{0}^{1}\left(R_{+}\right)$, $s>\frac{3}{2}$. Here $R_{+}=[0, \infty)$.

Let us now consider the following initial boundary value problem the DegasperisProcesi equation on the half line:

$$
\begin{cases}u_{t}-u_{t x x}+4 u u_{x}=3 u_{x} u_{x x}+u u_{x x x}, & t>0, x \in R_{+}, \\ u(0, x)=u_{0}(x), & x \in R_{+}, \\ u(t, 0)=0, & t \geq 0\end{cases}
$$

We first present the local well-posedness result for Eq.(3.1).

THEOREM 3.1. Assume that $u_{0} \in H^{s}\left(R_{+}\right) \cap H_{0}^{1}\left(R_{+}\right)$with $\frac{3}{2}<s \leq 2$. Then there exists a maximal $T=T\left(u_{0}\right)>0$, and a unique solution $u(t, x)$ to Eq.(3.1) such that $u=u\left(\cdot, u_{0}\right)$ belongs to

$$
C\left([0, T) ; H^{s}\left(R_{+}\right) \cap H_{0}^{1}\left(R_{+}\right)\right) \cap C^{1}\left([0, T) ; H^{s-1}\left(R_{+}\right) \cap H_{0}^{1}\left(R_{+}\right)\right) .
$$

Moreover, the solution depends continuously on the initial data, i.e. the mapping $u_{0} \mapsto$ $u\left(\cdot, u_{0}\right): H^{s}\left(R_{+}\right) \cap H_{0}^{1}\left(R_{+}\right) \rightarrow C\left([0, T) ; H^{s}\left(R_{+}\right) \cap H_{0}^{1}\left(R_{+}\right)\right) \cap C^{1}\left([0, T) ; H^{s-1}\left(R_{+}\right) \cap\right.$ $\left.H_{0}^{1}\left(R_{+}\right)\right)$is continuous. Furthermore, the maximal $T$ is independent of $s$ in the following sense: If the solution $u=u\left(\cdot, u_{0}\right)$ to Eq.(3.1) satisfies (3.2) and if $u_{0} \in H^{s^{\prime}}\left(R_{+}\right) \cap H_{0}^{1}\left(R_{+}\right)$ with $\frac{3}{2}<s \leq s^{\prime} \leq 2$, then

$$
u \in C\left([0, T) ; H^{s^{\prime}}\left(R_{+}\right) \cap H_{0}^{1}\left(R_{+}\right)\right) \cap C^{1}\left([0, T) ; H^{s^{\prime}-1}\left(R_{+}\right) \cap H_{0}^{1}\left(R_{+}\right)\right)
$$

with the same $T$.

Proof. We first convert the initial boundary value problem of Eq.(3.1) into the Cauchy problem of the Degasperis-Procesi equation on the line. In order to do so, we extend the initial data $u_{0}(x)$ defined on the half line into an odd function defined on the line:

$$
\tilde{u}_{0}(x)= \begin{cases}u_{0}(x), & x \geq 0, \\ -u_{0}(-x), & x<0 .\end{cases}
$$


Note that $u_{0}(x) \in H^{s}\left(R_{+}\right) \cap H_{0}^{1}\left(R_{+}\right)$with $\frac{3}{2}<s \leq 2$. The relation (3.3) and Lemma 2.3 shows that $\tilde{u}_{0}(x) \in H^{s}(R)$ with $\frac{3}{2}<s \leq 2$ is an odd function.

We now can convert Eq.(3.1) into the Degasperis-Procesi equation on the whole line.

$$
\left\{\begin{array}{lc}
\tilde{u}_{t}-\tilde{u}_{t x x}+4 \tilde{u} \tilde{u}_{x}=3 \tilde{u}_{x} \tilde{u}_{x x}+\tilde{u} \tilde{u}_{x x x}, & t>0, x \in R, \\
\tilde{u}(0, x)=\tilde{u}_{0}(x) \quad \text { (odd), } & x \in R .
\end{array}\right.
$$

Note that if $p(x):=\frac{1}{2} e^{-|x|}, x \in R$, then $\left(1-\partial_{x}^{2}\right)^{-1} f=p * f$ for all $f \in L^{2}(R)$ and $p *\left(\tilde{u}-\tilde{u}_{x x}\right)=\tilde{u}$. Using this identity, we can rewrite Eq.(3.4) as a quasi-linear evolution equation of hyperbolic type:

$$
\begin{cases}\tilde{u}_{t}+\tilde{u} \tilde{u}_{x}+\partial_{x} p *\left(\frac{3}{2} \tilde{u}^{2}\right)=0, & t>0, x \in R, \\ \tilde{u}(0, x)=\tilde{u}_{0}(x) \quad(\text { odd }), & x \in R .\end{cases}
$$

Applying the previous local well-posedness result of the Cauchy problem for the Degasperis-Procesi equation on the line [44], we have that there exists a maximal $T=T\left(\tilde{u}_{0}\right)>$ 0 , and a unique solution $\tilde{u}(t, x)$ to Eq.(3.5) such that

$$
\tilde{u}=\tilde{u}\left(\cdot, \tilde{u}_{0}\right) \in C\left([0, T) ; H^{s}(R)\right) \cap C^{1}\left([0, T) ; H^{s-1}(R)\right) .
$$

Moreover, the solution depends continuously on the initial data, i.e. the mapping $\tilde{u}_{0} \mapsto$ $\left.\tilde{u}\left(\cdot, \tilde{u}_{0}\right): H^{s}(R)\right) \rightarrow C\left([0, T) ; H^{s}(R)\right) \cap C^{1}\left([0, T) ; H^{s-1}(R)\right)$ is continuous. Furthermore, the maximal $T$ is independent of $s$ in the following sense: If the solution $\tilde{u}=\tilde{u}\left(\cdot, \tilde{u}_{0}\right)$ to Eq. (3.5) is in $C\left([0, T) ; H^{s}(R)\right) \cap C^{1}\left([0, T) ; H^{s-1}(R)\right)$ and if $\tilde{u}_{0} \in H^{s^{\prime}}(R)$ with $\frac{3}{2}<s \leq$ $s^{\prime} \leq 2$, then $\tilde{u} \in C\left([0, T) ; H^{s^{\prime}}(R)\right) \cap C^{1}\left([0, T) ; H^{s^{\prime}-1}(R)\right)$ with the same $T$.

Moreover, if $\tilde{u}(t, x)$ is a solution to Eq.(3.5), then one can check that the function $\tilde{u}_{1}(t, x):=-\tilde{u}(t,-x),(t, x) \in[0, T) \times R$ is also a solution of Eq.(3.5) in $C\left([0, T) ; H^{s}(R)\right) \cap$ $C^{1}\left([0, T) ; H^{s-1}(R)\right)$ with the initial data $\tilde{u}_{0}$. By uniqueness we conclude that $\tilde{u}_{1} \equiv \tilde{u}$. Since $\tilde{u}_{0}(x)$ is odd, it follows that $\tilde{u}(t, x)$ is odd for any $t \in[0, T)$. Therefore, we have $\tilde{u}(t, 0) \equiv 0$ for all $t \in[0, T)$.

Set $u(t, x)=r \tilde{u}(t, x)$ for all $(t, x) \in[0, T) \times R_{+}$. Then we know from Lemma 2.2 that $u(t, x) \in C\left([0, T) ; H^{s}\left(R_{+}\right) \cap H_{0}^{1}\left(R_{+}\right)\right) \cap C^{1}\left([0, T) ; H^{s-1}\left(R_{+}\right) \cap H_{0}^{1}\left(R_{+}\right)\right)$is a solution to Eq.(3.1). On the other hand, if $v(t, x)$ is also a solution to Eq.(3.1) with the same initial data $u_{0}(x)$, then

$$
\tilde{v}(t, x)= \begin{cases}v(t, x), & x \geq 0 \\ -v(t,-x), & x<0\end{cases}
$$

is the unique solution satisfying (3.2) to Eq.(3.5) with the initial data $\tilde{u}_{0}(x)$. By the uniqueness, we know that $u(t, x)=v(t, x)$ is the unique solution to Eq.(3.1) with the initial data $u_{0}(x)$. Moreover, we can obtain that the solution $u(t, x)$ depends continuously on the initial data $u_{0}(x)$ and the maximal $T$ is independent of $s$. This completes the proof of the theorem.

REMARK 3.1. Assume that $u_{0} \in H^{s}\left(R_{+}\right) \cap H_{0}^{1}\left(R_{+}\right)$with $s>2$. Following the similar argument in Theorem 3.1, one cannot prove generally the corresponding solution $u \in$ $C\left([0, T) ; H^{s}\left(R_{+}\right) \cap H_{0}^{1}\left(R_{+}\right)\right) \cap C^{1}\left([0, T) ; H_{0}^{1}\left(R_{+}\right)\right)$, and can only obtain that there exists a maximal $T=T\left(u_{0}\right)>0$, and a unique solution $u(t, x)$ to Eq.(3.1) such that $u=u\left(\cdot, u_{0}\right)$ belongs to

$$
C\left([0, T) ; H^{2}\left(R_{+}\right) \cap H_{0}^{1}\left(R_{+}\right)\right) \cap C^{1}\left([0, T) ; H_{0}^{1}\left(R_{+}\right)\right) .
$$


In order to obtain more regular solution, we may consider the following initial boundary value problem:

$$
\left\{\begin{array}{lc}
u_{t}-u_{t x x}+4 u u_{x}=3 u_{x} u_{x x}+u u_{x x x}, & t>0, x \in R_{+}, \\
u(0, x)=u_{0}(x), & x \in R_{+}, \\
u^{(2 k)}(t, 0)=u^{(2 k-2)}(t, 0)=\cdots=u(t, 0)=0, t \geq 0 .
\end{array}\right.
$$

We now present the following more regular local well-posedness result.

Theorem 3.2. Assume that $u_{0} \in D^{s}\left(R_{+}\right)$with $2 k+\frac{1}{2}<s \leq 2 k+2$. Then there exists a maximal $T=T\left(u_{0}\right)>0$, and a unique solution $u(t, x)$ to Eq.(3.7) such that $u=u\left(\cdot, u_{0}\right) \in C\left([0, T) ; D^{s}\left(R_{+}\right)\right) \cap C^{1}\left([0, T) ; D^{s-1}\left(R_{+}\right)\right)$. Moreover, the solution depends continuously on the initial data, i.e. the mapping $u_{0} \mapsto u\left(\cdot, u_{0}\right): D^{s}(R) \rightarrow$ $C\left([0, T) ; D^{s}\left(R_{+}\right)\right) \cap C^{1}\left([0, T) ; D^{s-1}\left(R_{+}\right)\right)$is continuous. Furthermore, the maximal $T$ is independent of $s$ in the following sense: If the solution

$$
u=u\left(\cdot, u_{0}\right) \in C\left([0, T) ; D^{s}\left(R_{+}\right)\right) \cap C^{1}\left([0, T) ; D^{s-1}\left(R_{+}\right)\right)
$$

is the solution to (3.7) and if $u_{0} \in D^{s^{\prime}}\left(R_{+}\right)$with $2 k+\frac{1}{2}<s \leq s^{\prime} \leq 2 k+2$, then $u \in C\left([0, T) ; D^{s^{\prime}}\left(R_{+}\right)\right) \cap C^{1}\left([0, T) ; D^{s^{\prime}-1}\left(R_{+}\right)\right)$with the same $T$.

Proof. Following the argument in Theorem 3.1, we first extend the initial data $u_{0}(x)$ defined on the half line to an odd function $\tilde{u}_{0}(x)$ defined in (3.3) on the line. Since $u_{0} \in D^{s}\left(R_{+}\right)$, Lemma 2.4 implies that $\tilde{u}_{0}(x) \in H^{s}(R)$ is an odd function. Then, following the proof in Theorem 3.1, we can obtain the desired result of the theorem.

We then present a precise blow-up scenario of strong solutions to Eq.(3.1).

THEOREM 3.3. Given $u_{0} \in H^{s}\left(R_{+}\right) \cap H_{0}^{1}\left(R_{+}\right)$with $\frac{3}{2}<s \leq 2$, blow up of the solution $u=u\left(\cdot, u_{0}\right)$ to Eq.(3.1) in finite time $T<+\infty$ occurs if and only if

$$
\liminf _{t \uparrow T}\left\{\inf _{x \in R_{+}}\left[u_{x}(t, x)\right]\right\}=-\infty .
$$

Proof. As we did before, we first extend the initial data $u_{0}(x)$ defined on the half line into an odd function $\tilde{u}_{0}(x)$ defined in (3.3) on the line. By Theorem 3.1, we can obtain the odd solution $\tilde{u}(t, x)$ which is the corresponding strong solution to Eq.(3.5) with the initial data $\tilde{u}_{0}(x)$. Moreover, $u(t, x)=r \tilde{u}(t, x)$ is the unique strong solution to Eq.(3.5) with the initial data $u_{0}(x)$.

For the Degasperis-Procesi equation on the line [44], blow up of the solution $\tilde{u}=$ $\tilde{u}\left(\cdot, \tilde{u}_{0}\right)$ to Eq. $(3.5)$ in finite time $T<+\infty$ occurs if and only if

$$
\liminf _{t \uparrow T}\left\{\inf _{x \in R}\left[\tilde{u}_{x}(t, x)\right]\right\}=-\infty .
$$

Since $\tilde{u}(t, \cdot)$ is odd, it follows that $\tilde{u}_{x}(t, \cdot)$ is even. Thus, we have that

$$
\liminf _{t \uparrow T}\left\{\inf _{x \in R}\left[\tilde{u}_{x}(t, x)\right]\right\}=\liminf _{t \uparrow T}\left\{\inf _{x \in R_{+}}\left[u_{x}(t, x)\right]\right\} .
$$

The above two relations imply the desired result of the theorem.

We now present two blow-up results and one global existence result for Eq.(3.5). 
Theorem 3.4. Let $\varepsilon>0$ and $u_{0} \in H^{s}\left(R_{+}\right) \cap H_{0}^{1}\left(R_{+}\right)$with $\frac{3}{2}<s \leq 2$. Assume that there exists $x_{0} \in R_{+}$such that

$$
u_{0}^{\prime}\left(x_{0}\right)<-\frac{(1+\varepsilon) \sqrt{6}}{4}\left(\left\|u_{0}\right\|_{L^{\infty}}+\left(4 \sqrt{6}\left\|u_{0}\right\|_{L^{2}}^{2} \ln \left(1+\frac{2}{\varepsilon}\right)+\left\|u_{0}\right\|_{L_{\infty}^{2}}\right)^{\frac{1}{2}}\right) .
$$

Then the corresponding solution to Eq.(3.1) blows up in finite time.

Proof. As we mentioned before, we may extend the initial data $u_{0}(x)$ defined on the half line to an odd function $\tilde{u}_{0}(x)$ defined in (3.3) on the line. By Theorem 3.1, we can obtain the odd solution $\tilde{u}(t, x)$ which is the corresponding strong solution to Eq.(3.5) with the initial data $\tilde{u}_{0}(x)$. Moreover, $u(t, x)=r \tilde{u}(t, x)$ is the unique strong solution to Eq.(3.1) with the initial data $u_{0}(x)$.

For the Degasperis-Procesi equation on the line, if there exists $x_{1} \in R$ such that

$$
\tilde{u}_{0}^{\prime}\left(x_{0}\right)<-\frac{(1+\varepsilon) \sqrt{6}}{4}\left(\left\|\tilde{u}_{0}\right\|_{L^{\infty}}+\left(2 \sqrt{6}\left\|\tilde{u}_{0}\right\|_{L^{2}}^{2} \ln \left(1+\frac{2}{\varepsilon}\right)+\left\|\tilde{u}_{0}\right\|_{L_{\infty}^{2}}\right)^{\frac{1}{2}}\right),
$$

then the corresponding solution to Eq.(3.5) blows up in finite time, see [31]. Since $\tilde{u}(t, \cdot)$ is odd, in view of Theorem 3.3 and (3.8), it follows that there exists $x_{0} \in R_{+}$such that

$$
\begin{aligned}
\tilde{u}_{0}^{\prime}\left(x_{0}\right)=u_{0}^{\prime}\left(x_{0}\right) & <-\frac{(1+\varepsilon) \sqrt{6}}{4}\left(\left\|u_{0}\right\|_{L^{\infty}}+\left(4 \sqrt{6}\left\|u_{0}\right\|_{L^{2}}^{2} \ln \left(\left(1+\frac{2}{\varepsilon}\right)+\left\|u_{0}\right\|_{L_{\infty}^{2}}\right)^{\frac{1}{2}}\right)\right. \\
& =-\frac{(1+\varepsilon) \sqrt{6}}{4}\left(\left\|\tilde{u}_{0}\right\|_{L^{\infty}}+\left(2 \sqrt{6}\left\|\tilde{u}_{0}\right\|_{L^{2}}^{2} \ln \left(\left(1+\frac{2}{\varepsilon}\right)+\left\|\tilde{u}_{0}\right\|_{L_{\infty}^{2}}\right)^{\frac{1}{2}}\right) .\right.
\end{aligned}
$$

Thus, the corresponding solution $u(t, x)$ to Eq.(3.1) blows up in finite time.

Theorem 3.5. Let $u_{0} \in H^{s}\left(R_{+}\right) \cap H_{0}^{1}\left(R_{+}\right)$with $\frac{3}{2}<s \leq 2$ and $y_{0}(x):=u_{0}(x)-u_{0, x x}(x)$. Assume that $y_{0}(x) \not \equiv 0$ and $y_{0}(x) \leq 0$ for all $x \in R_{+}$. Then the corresponding solution to Eq.(3.1) blows up in finite time.

Proof. Let $\tilde{u}_{0}(x)$ is defined in $(3.3)$ and set $\tilde{y}_{0}(x):=\tilde{u}_{0}(x)-\tilde{u}_{0, x x}(x)$. For the DegasperisProcesi equation on the line, if we assume that $\tilde{y}_{0}(x) \geq 0$ for all $x \in R_{-}, \tilde{y}_{0}(x) \leq 0$ for all $x \in R_{+}$, and $\tilde{y}_{0}(x)$ changes sign, then the corresponding solution $\tilde{u}(t, x)$ to Eq.(3.5) blows up in finite time, see [31].

Since $\tilde{u}(t, \cdot)$ is odd, the assumptions of the theorem ensure that $\tilde{y}_{0}(x) \geq 0$ for all $x \in R_{-}, \tilde{y}_{0}(x) \leq 0$ for all $x \in R_{+}$, and $\tilde{y}_{0}(x)$ changes sign. Thus, by Theorem 2.3 and (3.8), the corresponding solution $u(t, x)$ to Eq.(3.1) blows up in finite time.

REMARK 3.2. Note that if $y_{0}(x) \not \equiv 0$ and $y_{0}(x) \leq 0$ for all $x \in R$, then the corresponding solution to the Degasperis-Procesi equation on the whole line exists globally in time, see [31]. Theorem 3.5 shows that there is a considerable difference between the DegasperisProcesi equation on the whole line and the case of the half line.

Theorem 3.6. Let $u_{0} \in H^{s}\left(R_{+}\right) \cap H_{0}^{1}\left(R_{+}\right)$with $\frac{3}{2}<s \leq 2$ and $y_{0}(x):=u_{0}(x)-u_{0, x x}(x)$. Assume that $y_{0}(x) \geq 0$ for all $x \in R_{+}$. Then the corresponding solution to Eq.(3.1) exists globally in time.

Proof. Let $\tilde{u}_{0}(x)$ is defined in (3.3) and set $\tilde{y}_{0}(x):=\tilde{u}_{0}(x)-\tilde{u}_{0, x x}(x)$. For the DegasperisProcesi equation on the line, if we assume that $\tilde{y}_{0}(x) \leq 0$ for all $x \in R_{-}$and $\tilde{y}_{0}(x) \geq 0$ 
for all $x \in R_{+}$, then the corresponding solution $\tilde{u}(t, x)$ to Eq.(3.5) exists globally in time, see $[31]$.

Since $\tilde{u}(t, \cdot)$ is odd, the assumptions of the theorem ensure that $\tilde{y}_{0}(x) \leq 0$ for all $x \in R_{-}$and $\tilde{y}_{0}(x) \geq 0$ for all $x \in R_{+}$. Thus, by Theorem 3.3 and (3.8), we see that the corresponding solution $u(t, x)$ to Eq.(3.1) exists globally in time.

REMARK 3.3. Given $u_{0} \in D^{s}\left(R_{+}\right)$with $2 k+\frac{1}{2}<s \leq 2 k+2$ and $k \in N \backslash\{0\}$. Theorems 3.3-3.6 hold true for the corresponding solution $u(t, x)$ to Eq.(3.7).

Let us now turn to weak solutions to Eq.(3.1). Set

$$
h(x, \xi):=\frac{1}{2}\left(e^{-|x-\xi|}-e^{-|x+\xi|}\right), \quad \forall x, \xi \in R_{+} .
$$

Then, the boundary value problem to the elliptic equation

$$
\left\{\begin{array}{l}
v(x)-v_{x x}(x)=f(x), x \in R_{+} \\
v(0)=v(+\infty)=0
\end{array}\right.
$$

has a unique solution $v(x)=\int_{0}^{\infty} h(x, \xi) f(\xi) d \xi$ in $H^{2}\left(R_{+}\right) \cap H_{0}^{1}\left(R_{+}\right)$provided $f$ belongs to $L^{2}\left(R_{+}\right)$. Thus, Eq.(3.1) is formally equivalent to the following initial value problem for the Degasperis-Procesi equation on the half line:

$$
\left\{\begin{array}{lc}
u_{t}+u u_{x}+\frac{3}{2} \partial_{x} \int_{0}^{\infty} h(x, \xi) u^{2}(t, \xi) d \xi=0, & t>0, x>0, \\
u(0, x)=u_{0}(x), & x \in R_{+} .
\end{array}\right.
$$

Take

$$
F(u)=\frac{u^{2}}{2}+\frac{3}{2} \int_{0}^{\infty} h(x, \xi) u^{2}(t, \xi) d \xi .
$$

Then, Eq.(3.9) can be rewritten as the conservation law

$$
u_{t}+F(u)_{x}=0, \quad u(0, x)=u_{0}, \quad t>0, x \in R_{+} .
$$

We now introduce the notion of weak solutions to Eq.(3.10). For this let $C_{c}^{\infty}\left([0, T) \times R_{+}\right)$ denote the space of all functions on $[0, T) \times R_{+}$, which may be obtained as the restriction to $[0, T) \times R_{+}$of a smooth function on $R^{2}$ with compact support contained in $(-T, T) \times R_{+}$.

Definition 3.1. Let $u_{0} \in H_{0}^{1}\left(R_{+}\right)$. If $u \in L_{l o c}^{\infty}\left([0, T) ; H_{0}^{1}\left(R_{+}\right)\right)$satisfies the following identity

$$
\int_{0}^{T} \int_{R_{+}}\left(u \psi_{t}+F(u) \psi_{x}\right) d x d t+\int_{R_{+}} u_{0}(x) \psi(0, x) d x=0
$$

for all $\psi \in C_{c}^{\infty}\left([0, T) \times R_{+}\right)$, then $u$ is called a weak solution to Eq.(3.10). If $u$ is a weak solution on $[0, T)$ for every $T>0$, then it is called global weak solution to Eq.(3.10) (or to Eq.(3.1)).

ThEOREM 3.7. Assume that $u_{0} \in H_{0}^{1}\left(R_{+}\right)$with $y_{0}:=u_{0}-u_{0, x x} \in M^{+}\left(R_{+}\right)$. Then Eq.(3.1) has a unique global weak solution

$$
u \in W_{l o c}^{1, \infty}\left(R_{+} \times R_{+}\right) \cap L_{l o c}^{\infty}\left(R_{+} ; H^{1}\left(R_{+}\right)\right)
$$

with the initial data $u_{0}$. Moreover, $E(u)=\int_{R_{+}} y\left(4-\partial_{x}^{2}\right)^{-1} u d x$ is a conservation law. 
Proof. As we mentioned before, we can extend the initial data $u_{0}(x)$ defined on the half line to an odd function $\tilde{u}_{0}(x)$ defined in (3.3) on the line. Since $u_{0} \in H_{0}^{1}\left(R_{+}\right)$, it follows that $\tilde{u}_{0} \in H^{1}(R)$.

By assumption of the theorem and the odd property of $\tilde{u}(t, x)$, we have that

$$
\operatorname{supp}\left\{\tilde{y}_{0}^{+}\right\} \subset[0, \infty) \text { and } \operatorname{supp}\left\{\tilde{y}_{0}^{-}\right\} \subset(-\infty, 0] .
$$

For the Degasperis-Procesi equation on the line, if $\operatorname{supp}\left\{\tilde{y}_{0}^{+}\right\} \subset[0, \infty)$ and $\operatorname{supp}\left\{\tilde{y}_{0}^{-}\right\} \subset$ $(-\infty, 0]$, then Eq.(3.5) has a unique global weak solution

$$
\tilde{u} \in W_{l o c}^{1, \infty}\left(R_{+} \times R\right) \cap L_{l o c}^{\infty}\left(R_{+} ; H^{1}(R)\right)
$$

with initial data $\tilde{u}_{0}$, cf. [20]. Moreover, $E(\tilde{u})=\int_{R} \tilde{y}\left(4-\partial_{x}^{2}\right)^{-1} \tilde{u} d x$ is a conservation law. Again, since $\tilde{u}(t, x)$ is odd for any $t \in R_{+}$, we obtain the desired result for $u(t, x)$.

4. The case of the interval $[0,1]$. In the section, we mainly study initial boundary value problems of the Degasperis-Procesi equation on the interval $[0,1]$ with initial data $u_{0} \in H^{s}([0,1]) \cap H_{0}^{1}([0,1]), s>\frac{3}{2}$.

Let us now consider the following initial boundary value problem for the DegasperisProcesi equation on the interval $[0,1]$ :

$$
\begin{cases}u_{t}-u_{t x x}+3 u u_{x}=2 u_{x} u_{x x}+u u_{x x x}, & t>0, x \in[0,1], \\ u(0, x)=u_{0}(x), & x \in[0,1], \\ u(t, 0)=u(t, 1)=0, & t \geq 0 .\end{cases}
$$

We first present the local well-posedness result for Eq.(4.1).

TheOrem 4.1. Assume that $u_{0} \in H^{s}([0,1]) \cap H_{0}^{1}([0,1])$ with $\frac{3}{2}<s \leq 2$. Then there exists a maximal $T=T\left(u_{0}\right)>0$, and a unique solution $u(t, x)$ to Eq.(4.1) such that $u=u\left(\cdot, u_{0}\right)$ belongs to

$$
C\left([0, T) ; H^{s}([0,1]) \cap H_{0}^{1}([0,1])\right) \cap C^{1}\left([0, T) ; H^{s-1}([0,1]) \cap H_{0}^{1}([0,1])\right) .
$$

Moreover, the solution depends continuously on the initial data, i.e. the mapping

$$
\begin{aligned}
u_{0} \mapsto u\left(\cdot, u_{0}\right): & H^{s}([0,1]) \cap H_{0}^{1}([0,1]) \rightarrow \\
& C\left([0, T) ; H^{s}([0,1]) \cap H_{0}^{1}([0,1])\right) \cap C^{1}\left([0, T) ; H^{s-1}([0,1]) \cap H_{0}^{1}([0,1])\right)
\end{aligned}
$$

is continuous. Furthermore, the maximal $T$ is independent of $s$ in the following sense: If the solution $u=u\left(\cdot, u_{0}\right)$ to Eq.(4.1) satisfies (4.2) and if $u_{0} \in H^{s^{\prime}}([0,1]) \cap H_{0}^{1}([0,1])$ with $\frac{3}{2}<s \leq s^{\prime} \leq 2$, then

$$
u \in C\left([0, T) ; H^{s^{\prime}}([0,1]) \cap H_{0}^{1}([0,1])\right) \cap C^{1}\left([0, T) ; H^{s^{\prime}-1}([0,1]) \cap H_{0}^{1}([0,1])\right)
$$

with the same $T$.

Proof. We first convert the initial boundary value problem of the Degasperis-Procesi equation on the interval $[0,1]$ into the Cauchy problem for the periodic Camassa-Holm equation with period 2 . In order to do so, we extend the initial data $u_{0}(x)$ defined on the interval $[0,1]$ to a periodic odd function defined on the line:

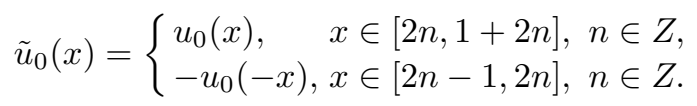


Note that $u_{0}(x) \in H^{s}([0,1]) \cap H_{0}^{1}([0,1])$ with $\frac{3}{2}<s \leq 2$. Then we have

$$
\begin{aligned}
\lim _{x \rightarrow 1^{-}} \frac{d \tilde{u}_{0}(x)}{d x} & =\lim _{x \rightarrow 1^{-}} \frac{\tilde{u}_{0}(x)-\tilde{u}_{0}(1)}{x-1}=\lim _{x \rightarrow 1^{-}} \frac{u_{0}(x)}{x-1}=\lim _{x \rightarrow(-1)^{+}} \frac{-u_{0}(-x)}{x+1} \\
& =\lim _{x \rightarrow(-1)^{+}} \frac{\tilde{u}_{0}(x)-\tilde{u}_{0}(-1)}{x-(-1)}=\lim _{x \rightarrow(-1)^{+}} \frac{d \tilde{u}_{0}(x)}{d x}=\lim _{x \rightarrow 1^{+}} \frac{d \tilde{u}_{0}(x)}{d x} .
\end{aligned}
$$

Combining the above relation with (4.3) and Lemma 2.6, we have that

$$
\tilde{u}_{0}(x) \in H^{s}([-1,1]) \cap H_{0}^{1}([-1,1])
$$

with $\frac{3}{2}<s \leq 2$ is a periodic odd function.

Thus, we may convert the Degasperis-Procesi equation on the interval $[0,1]$ into the following periodic case.

$$
\begin{cases}\tilde{u}_{t}-\tilde{u}_{t x x}+4 \tilde{u} \tilde{u}_{x}=3 \tilde{u}_{x} \tilde{u}_{x x}+\tilde{u} \tilde{u}_{x x x}, & t>0, x \in R, \\ \tilde{u}(0, x)=\tilde{u}_{0}(x) \quad(\text { odd }), & x \in R, \\ \tilde{u}_{0}(0)=\tilde{u}_{0}(1)=0, & t \geq 0, x \in R . \\ \tilde{u}(t, x)=\tilde{u}_{0}(t, x+2) & \end{cases}
$$

Applying the local well-posedness result for the periodic Degasperis-Procesi equation [45], we have that there exists a maximal $T=T\left(\tilde{u}_{0}\right)>0$, and a unique solution $\tilde{u}(t, x)$ to Eq.(4.4) such that $\tilde{u}=\tilde{u}\left(\cdot, \tilde{u}_{0}\right)$ belongs to

$$
C\left([0, T) ; H^{s}([-1,1]) \cap H_{0}^{1}([-1,1])\right) \cap C^{1}\left([0, T) ; H^{s-1}([-1,1]) \cap H_{0}^{1}([-1,1])\right) .
$$

Moreover, the solution depends continuously on the initial data, i.e. the mapping

$$
\begin{aligned}
\left.\left.\tilde{u}_{0} \mapsto \tilde{u}\left(\cdot, \tilde{u}_{0}\right): \quad H^{s}([-1,1])\right) \cap H_{0}^{1}([-1,1])\right) \rightarrow \\
\quad C\left([0, T) ; H^{s}([-1,1]) \cap H_{0}^{1}([-1,1])\right) \cap C^{1}\left([0, T) ; H^{s-1}([-1,1]) \cap H_{0}^{1}([-1,1])\right)
\end{aligned}
$$

is continuous. Furthermore, the maximal $T$ is independent of $s$ in the following sense: If the solution $\tilde{u}=\tilde{u}\left(\cdot, \tilde{u}_{0}\right)$ to Eq.(4.4) satisfies (4.5) and if $\left.\tilde{u}_{0} \in H^{s^{\prime}}([-1,1]) \cap H_{0}^{1}([-1,1])\right)$ with $\frac{3}{2}<s \leq s^{\prime} \leq 2$, then $\tilde{u}$ belongs to

$$
C\left([0, T) ; H^{s^{\prime}}([-1,1]) \cap H_{0}^{1}([-1,1])\right) \cap C^{1}\left([0, T) ; H^{s^{\prime}-1}([-1,1]) \cap H_{0}^{1}([-1,1])\right)
$$

with the same $T$.

Note that if $\tilde{u}(t, x)$ is a solution to Eq.(4.4), then one can check that the function $v(t, x):=-\tilde{u}(t,-x),(t, x) \in[0, T) \times R$ is also a solution of Eq.(4.4) satisfying (4.5) with the initial data $\tilde{u}_{0}$. By uniqueness we conclude that $v \equiv \tilde{u}$ and $\tilde{u}(t, \cdot)$ is odd for any $t \in[0, T)$. Therefore, we have $\tilde{u}(t, 0) \equiv 0$ for all $t \in[0, T)$.

Set $u(t, x)=r_{1} \tilde{u}(t, x)$ for all $(t, x) \in[0, T) \times[0,1]$. Then we know that

$$
u(t, x) \in C\left([0, T) ; H^{s}([0,1]) \cap H_{0}^{1}([0,1])\right) \cap C^{1}\left([0, T) ; H^{s-1}([0,1]) \cap H_{0}^{1}([0,1])\right)
$$

is a solution to Eq.(4.1). On the other hand, if $v(t, x)$ is a solution to Eq.(4.1) with the initial data $u_{0}(x)$, then

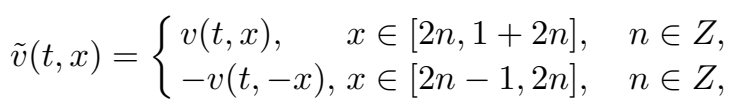

is the solution to Eq.(4.4) with the initial data $\tilde{u}_{0}(x)$ satisfying (4.5). By the uniqueness of $\tilde{u}(t, x)$, we know that $u(t, x)=v(t, x)$ is the unique solution to Eq.(4.1) with the initial 
data $u_{0}(x)$. Moreover, the solution $u(t, x)$ depends continuously on the initial data $u_{0}(x)$ and the maximal $T$ is independent of $s$. This completes the proof of the theorem.

REMARK 4.1. Assume that $u_{0} \in H^{s}([0,1]) \cap H_{0}^{1}([0,1])$ with $s>2$. Following the similar argument in Theorem 4.1, one cannot prove generally the corresponding solution $u \in$ $C\left([0, T) ; H^{s}([0,1]) \cap H_{0}^{1}([0,1])\right) \cap C^{1}\left([0, T) ; H_{0}^{1}([0,1])\right)$, and can only obtain that there exists a maximal $T=T\left(u_{0}\right)>0$, and a unique solution $u(t, x)$ to Eq.(4.1) such that $u=u\left(\cdot, u_{0}\right)$ belongs to

$$
C\left([0, T) ; H^{2}([0,1]) \cap H_{0}^{1}([0,1])\right) \cap C^{1}\left([0, T) ; H_{0}^{1}([0,1])\right) .
$$

In order to obtain the more regular solution, we may consider the following initial boundary value problem:

$$
\begin{cases}u_{t}-u_{t x x}+3 u u_{x}=2 u_{x} u_{x x}+u u_{x x x}, & t>0, x \in[0,1], \\ u(0, x)=u_{0}(x), & x \in[0,1], \\ u^{(2 k)}(t, 0)=u^{(2 k-2)}(t, 0)=\cdots=u(t, 0)=0, t \geq 0, \\ u^{(2 k)}(t, 1)=u^{(2 k-2)}(t, 1)=\cdots=u(t, 1)=0, t \geq 0 .\end{cases}
$$

We now present the following more regular local well-posedness result.

Theorem 4.2. Assume that $u_{0} \in D^{s}([0,1])$ with $2 k+\frac{1}{2}<s \leq 2 k+2$. Then there exists a maximal $T=T\left(u_{0}\right)>0$, and a unique solution $u(t, x)$ to Eq.(4.7) such that $u=$ $u\left(\cdot, u_{0}\right) \in C\left([0, T) ; D^{s}([0,1])\right) \cap C^{1}\left([0, T) ; D^{s-1}([0,1])\right)$. Moreover, the solution depends continuously on the initial data, i.e. the mapping

$$
u_{0} \mapsto u\left(\cdot, u_{0}\right): D^{s}([0,1]) \rightarrow C\left([0, T) ; D^{s}([0,1])\right) \cap C^{1}\left([0, T) ; D^{s-1}([0,1])\right)
$$

is continuous. Furthermore, the maximal $T$ is independent of $s$ in the following sense: If

$$
u=u\left(\cdot, u_{0}\right) \in C\left([0, T) ; D^{s}([0,1])\right) \cap C^{1}\left([0, T) ; D^{s-1}([0,1])\right)
$$

is the solution to Eq.(4.7) and if $u_{0} \in D^{s^{\prime}}([0,1])$ with $2 k+\frac{1}{2}<s \leq s^{\prime} \leq 2 k+2$, then $u \in C\left([0, T) ; D^{s^{\prime}}([0,1])\right) \cap C^{1}\left([0, T) ; D^{s^{\prime}-1}([0,1])\right)$ with the same $T$.

Proof. Following the argument of Theorem 4.1, we first extend the initial data $u_{0}(x)$ defined on the interval $[0,1]$ into an periodic odd function $\tilde{u}_{0}(x)$ defined in $(4.3)$ on the line. Since $u_{0} \in D^{s}([0,1])$, Lemma 2.7 and (4.3) show that $\tilde{u}_{0}(x) \in D^{s}([-1,1])$ is a periodic odd function. Then following the proof in Theorem 4.1, we can obtain the desired result of the theorem.

We then present a precise blow-up scenario of strong solutions to Eq.(4.1).

THEOREM 4.3. Given $u_{0} \in H^{s}([0,1]) \cap H_{0}^{1}([0,1])$ with $\frac{3}{2}<s \leq 2$, blow up of the solution $u=u\left(\cdot, u_{0}\right)$ to Eq.(4.1) in finite time $T<+\infty$ occurs if and only if

$$
\liminf _{t \uparrow T}\left\{\inf _{x \in[0,1]}\left[u_{x}(t, x)\right]\right\}=-\infty .
$$

Proof. As we did before, we first extend the initial data $u_{0}(x)$ defined on the interval $[0,1]$ into an periodic odd function $\tilde{u}_{0}(x)$ defined in (4.3) on the line. By Theorem 4.1, we can obtain the odd equation $\tilde{u}(t, x)$ which is the corresponding strong solution to Eq.(4.4) with the initial data $\tilde{u}_{0}(x)$. Moreover, $u(t, x)=r_{1} \tilde{u}(t, x)$ is the unique strong solution to Eq.(4.1) with the initial data $u_{0}(x)$. 
For the periodic Degasperis-Procesi equation [45], blow up of the solution $\tilde{u}=\tilde{u}\left(\cdot, \tilde{u}_{0}\right)$ to Eq.(4.4) in finite time $T<+\infty$ occurs if and only if

$$
\liminf _{t \uparrow T}\left\{\inf _{x \in[-1,1]}\left[\tilde{u}_{x}(t, x)\right]\right\}=-\infty .
$$

Since $\tilde{u}(t, \cdot)$ is odd, it follows that $\tilde{u}_{x}(t, \cdot)$ is even. Thus, we have that

$$
\liminf _{t \uparrow T}\left\{\inf _{x \in[-1,1]}\left[\tilde{u}_{x}(t, x)\right]\right\}=\liminf _{t \uparrow T}\left\{\inf _{x \in[0,1]}\left[u_{x}(t, x)\right]\right\} .
$$

The above two relations imply the desired result.

We now present a blow-up result for Eq.(4.1).

THEOREM 4.4. Assume that $u_{0} \in H^{s}([0,1]) \cap H_{0}^{1}([0,1])$ with $\frac{3}{2}<s \leq 2$ and $u_{0} \not \equiv 0$. Then the corresponding solution to Eq.(4.1) blows up in finite time.

Proof. As we mentioned before, we may extend the initial data $u_{0}(x)$ defined on the interval $[0,1]$ to a periodic odd function $\tilde{u}_{0}(x)$ defined in $(4.3)$ on the line. By Theorem 4.1 , we can obtain the periodic odd function $\tilde{u}(t, x)$ which is the corresponding strong solution to Eq.(4.4) with the initial data $\tilde{u}_{0}(x)$. Moreover, $u(t, x)=r_{1} \tilde{u}(t, x)$ is the unique strong solution to Eq.(4.1) with the initial data $u_{0}(x)$.

For the periodic Degasperis-Procesi equation, if $u_{0} \not \equiv 0$ and for any $t \in[0, T)$ there exists $x_{t}$ such that $\tilde{u}\left(t, x_{t}\right)=0$, then the corresponding solution to Eq.(4.1) blows up in finite time, see [20]. Since $\tilde{u}(t, \cdot)$ is odd, it follows that $\tilde{u}(t, 0)=0$. Thus, we deduce that the corresponding solution $\tilde{u}(t, x)$ blows up in finite time. By Theorem 4.3, we have that the corresponding solution $u(t, x)$ also blows up in finite time.

As a generalization of Theorem 4.4, we have the following result.

Theorem 4.5. Assume that $u_{0} \in D^{s}([0,1])$ with $2 k+\frac{1}{2}<s \leq 2 k+2$ and $k \in N \backslash\{0\}$, and $u_{0} \not \equiv 0$. Then the corresponding solution to Eq.(4.7) blows up in finite time.

Next, we study weak solutions to Eq.(4.1). For all $x, \xi \in R_{+}$we set

$$
G(x, \xi):=\left\{\begin{array}{l}
\frac{\sinh (x) \sinh (1-\xi)}{\sinh (1)}, 0 \leq x \leq \xi, \\
\frac{\sinh (\xi) \sinh (1-x)}{\sinh (1)}, \xi<x \leq 1 .
\end{array}\right.
$$

Then, the boundary value problem to the elliptic equation

$$
\left\{\begin{array}{l}
v(x)-v_{x x}(x)=f(x), x \in[0,1], \\
v(0)=v(1)=0
\end{array}\right.
$$

has a unique solution $v(x)=\int_{0}^{1} G(x, \xi) f(\xi) d \xi$ in $H^{2}([0,1]) \cap H_{0}^{1}([0,1])$ provided $f$ belongs to $L^{2}([0,1])$. Thus, Eq. $(4.1)$ is formally equivalent to the following initial value problem for the Degasperis-Procesi equation:

$$
\left\{\begin{array}{lc}
u_{t}+u u_{x}+\frac{3}{2} \partial_{x} \int_{0}^{1} G(x, \xi) u^{2}(t, \xi) d \xi=0, & t>0, x \in[0,1], \\
u(0, x)=u_{0}(x), & x \in[0,1] .
\end{array}\right.
$$

Take $F(u)=\frac{u^{2}}{2}+\frac{3}{2} \int_{0}^{1} G(x, \xi) u^{2}(t, \xi) d \xi$. Then, Eq.(4.9) can be rewritten as the conservation law

$$
u_{t}+F(u)_{x}=0, \quad u(0, x)=u_{0}, \quad t>0, x \in[0,1]
$$


We now introduce the notion of weak solutions to Eq.(4.10). For this let $C_{c}^{\infty}([0, T) \times$ $[0,1])$ denote the space of all functions on $[0, T) \times[0,1]$, which may be obtained as the restriction to $[0, T) \times[0,1]$ of a smooth function on $R^{2}$ with compact support contained in $(-T, T) \times[0,1]$.

Definition 4.1. Let $u_{0} \in H_{0}^{1}([0,1])$. If $u \in L_{l o c}^{\infty}\left([0, T) ; H_{0}^{1}([0,1])\right)$ satisfies the following identity

$$
\int_{0}^{T} \int_{0}^{1}\left(u \psi_{t}+F(u) \psi_{x}\right) d x d t+\int_{0}^{1} u_{0}(x) \psi(0, x) d x=0
$$

for all $\psi \in C_{c}^{\infty}([0, T) \times[0,1])$, then $u$ is called a weak solution to Eq.(4.10). If $u$ is a weak solution on $[0, T)$ for every $T>0$, then it is called a global weak solution to Eq.(4.10) (or to Eq.(4.1)).

Theorem 4.6. Assume that $u_{0} \in H_{0}^{1}([0,1])$ with $y_{0}:=u_{0}-u_{0, x x} \in M([0,1])$. Then there exists $T=T\left(\left\|y_{0}\right\|_{M([0,1])}\right)>0$ and a unique solution to Eq.(4.1),

$$
u \in W_{l o c}^{1, \infty}\left(R_{+} \times[0,1]\right) \cap L_{l o c}^{\infty}\left(R_{+} ; H^{1}([0,1])\right)
$$

with $u_{0}$ as initial data.

Proof. As we mentioned before, we can extend the initial data $u_{0}(x)$ defined on the interval $[0,1]$ to a periodic odd function $\tilde{u}_{0}(x)$ defined in $(3.3)$ on the line with period 2. Since $u_{0} \in H_{0}^{1}([0,1])$, it follows that $\tilde{u}_{0} \in H_{0}^{1}([-1,1])$.

Following the proof of Theorem 4 in [34], we can also prove that if that $\tilde{u}_{0} \in$ $H^{1}([-1,1])$ with $\tilde{y}_{0}:=\tilde{u}_{0}-\tilde{u}_{0, x x} \in M([-1,1])$, then there exists $T=T\left(\left\|\tilde{y}_{0}\right\|_{M([-1,1])}\right)>0$ and a unique solution to Eq.(4.1),

$$
u \in W_{l o c}^{1, \infty}\left(R_{+} \times[0,1]\right) \cap L_{l o c}^{\infty}\left(R_{+} ; H^{1}([0,1])\right)
$$

with $\tilde{u}_{0}$ as initial data. Note that $\tilde{u}_{0}(x)$ is odd. By the uniqueness of $\tilde{u}(t, x)$, we can deduce that $\tilde{u}(t, \cdot)$ is odd for any $t \in[0, T)$. Thus, we can obtain the desired result for $u(t, x)$.

Acknowledgments. The authors thank the referee for valuable comments and suggestions.

\section{References}

[1] R. Beals, D. Sattinger, and J. Szmigielski, Acoustic scattering and the extended Kortewegde Vries hierarchy, Adv. Math. 140 (1998), 190-206.

[2] A. Bressan and A. Constantin, Global conservative solutions of the Camassa-Holm equation, Arch. Rat. Mech. Anal. 183 (2007), 215-239.

[3] R. Camassa and D. Holm, An integrable shallow water equation with peaked solitons, Phys. Rev. Letters 71 (1993), 1661-1664.

[4] R. Camassa, D. Holm and J. Hyman, A new integrable shallow water equation, Adv. Appl. Mech. 31 (1994), 1-33.

[5] G. M. Coclite and K. H. Karlsen, On the well-posedness of the Degasperis-Procesi equation, J. Funct. Anal. 233 (2006), 60-91. 
[6] G. M. Coclite, K. H. Karlsen and N. H. Risebro, Numerical schemes for computing discontinuous solutions of the Degasperis-Procesi equation, IMA J. Numer. Anal. 28 (2008), 80-105.

[7] A. Constantin, Global existence of solutions and breaking waves for a shallow water equation: a geometric approach, Ann. Inst. Fourier (Grenoble) 50 (2000), 321-362.

[8] A. Constantin, Finite propagation speed for the Camassa-Holm equation, J. Math. Phys. 46 (2005), 023506, 4 pp.

[9] A. Constantin and J. Escher, Global existence and blow-up for a shallow water equation, Annali Sc. Norm. Sup. Pisa 26 (1998), 303-328.

[10] A. Constantin and J. Escher, Well-posedness, global existence, and blowup phenomena for a periodic quasi-linear hyperbolic equation, Comm. Pure Appl. Math. 51 (1998), 475-504.

[11] A. Constantin and J. Escher, Wave breaking for nonlinear nonlocal shallow water equations, Acta Mathematica 181 (1998), 229-243.

[12] A. Constantin and B. Kolev, Geodesic flow on the diffeomorphism group of the circle, Comment. Math. Helv. 78 (2003), 787-804.

[13] A. Constantin and H. P. McKean, A shallow water equation on the circle, Comm. Pure Appl. Math. 52 (1999), 949-982.

[14] A. Constantin and L. Molinet, Global weak solutions for a shallow water equation, Comm. Math. Phys. 211 (2000), 45-61.

[15] A. Constantin and W. A. Strauss, Stability of peakons, Comm. Pure Appl. Math. 53 (2000), 603-610.

[16] A. Degasperis, D. D. Holm, and A. N. W. Hone, A new integral equation with peakon solutions, Theoretical and Mathematical Physics 133 (2002), 1463-1474.

[17] A. Degasperis, M. Procesi, Asymptotic integrability, in: Symmetry and Perturbation Theory, A. Degasperis and G. Gaeta (eds.), World Scientific, 1999, 23-37.

[18] H. R. Dullin, G. A. Gottwald, and D. D. Holm, An integrable shallow water equation with linear and nonlinear dispersion, Phys. Rev. Letters 87 (2001), 4501-4504.

[19] H. R. Dullin, G. A. Gottwald, and D. D. Holm, Camassa-Holm, Korteweg-de Vries-5 and other asymptotically equivalent equations for shallow water waves, Fluid Dynamics Research 33 (2003), 73-79.

[20] J. Escher, Y. Liu, and Z. Yin, Global weak solutions and blow-up structure for the Degasperis-Procesi equation, J. Funct. Anal., 241 (2006), 457-485.

[21] J. Escher, Y. Liu, and Z. Yin, Shock waves and blow-up phenomena for the periodic Degasperis-Procesi equation, Indiana Univ. Math. J. 56 (2007), 87-117.

[22] J. Escher and Z. Yin, Initial boundary value problems of the Camassa-Holm equation, Commun. Partial Differential Equations 33 (2008), 1-19.

[23] A. Fokas and B. Fuchssteiner, Symplectic structures, their Bäcklund transformation and hereditary symmetries, Physica D 4 (1981), 47-66.

[24] D. Gilbarg and N. S. Trudinger, Elliptic Partial Differential Equations of Second Order, Springer, Berlin, 1977.

[25] D. Henry, Infinite propagation speed for the Degasperis-Procesi equation, J. Math. Anal. Appl. 311 (2005), 755-759.

[26] D. D. Holm and M. F. Staley, Wave structure and nonlinear balances in a family of evolutionary PDEs, SIAM J. Appl. Dyn. Syst. 2 (2003), 323-380.

[27] R. S. Johnson, Camassa-Holm, Korteweg-de Vries and related models for water waves, J. Fluid Mech. 455 (2002), 63-82.

[28] K. H. Kwek, H. Gao, W. Zhang, and C. Qu, An initial boundary value problem of CamassaHolm equation, J. Math. Phys., 41 (2000), 8279-8285. 
[29] C. Kenig, G. Ponce, and L. Vega, Well-posedness and scattering results for the generalized Korteweg-de Veris equation via the contraction principle, Comm. Pure Appl. Math. 46 (1993), 527-620.

[30] J. Lenells, Traveling wave solutions of the Degasperis-Procesi equation, J. Math. Anal. Appl. 306 (2005), 72-82.

[31] Y. Liu and Z. Yin, Global existence and blow-up phenomena for the Degasperis-Procesi equation, Comm. Math. Phys. 267 (2006), 801-820.

[32] H. Lundmark, Formation and dynamics of shock waves in the Degasperis-Procesi equation, J. Nonlinear Science 17 (2007), 169-198.

[33] H. Lundmark and J. Szmigielski, Multi-peakon solutions of the Degasperis-Procesi equation, Inverse Problems 19 (2003), 1241-1245.

[34] L. Molinet, On well-posedness results for Camassa-Holm equation on the line: a survey, J. Nonlinear Math. Phys. 11 (2004), 521-533.

[35] S. Ma and S. Ding, On the initial boundary value problem for a shallow water equation, J. Math. Phys. 45 (2004), 3479-3497.

[36] Y. Matsuno, Multisoliton solutions of the Degasperis-Procesi equation and their peakon limit, Inverse Problems 21 (2005), 1553-1570.

[37] H. P. McKean, Integrable systems and algebraic curves, in: Global Analysis, Lecture Notes in Mathematics 755, Springer, 1979, 83-200.

[38] G. Misiolek, A shallow water equation as a geodesic flow on the Bott-Virasoro group, J. Geom. Phys. 24 (1998), 203-208.

[39] G. Rodriguez-Blanco, On the Cauchy problem for the Camassa-Holm equation, Nonlinear Anal. 46 (2001), 309-327.

[40] T. Tao, Low-regularity global solutions to nonlinear dispersive equations, in: Surveys in Analysis and Operator Theory (Canberra, 2001), Proc. Centre Math. Appl. Austral. Nat. Univ., 40, Austral. Nat. Univ. Canberra, 2002, 19-48.

[41] H. Triebel, Interpolation Theory, Function Spaces, Differential Operators, North-Holland, 1978.

[42] G. B. Whitham, Linear and Nonlinear Waves, Wiley, New York, 1980.

[43] Z. Xin and P. Zhang, On the weak solutions to a shallow water equation, Comm. Pure Appl. Math. 53 (2000), 1411-1433.

[44] Z. Yin, On the Cauchy problem for an integrable equation with peakon solutions, Illinois J. Math. 47 (2003), 649-666.

[45] Z. Yin, Global existence for a new periodic integrable equation, J. Math. Anal. Appl. 283 (2003), 129-139.

[46] Z. Yin, Global weak solutions to a new periodic integrable equation with peakon solutions, J. Funct. Anal. 212 (2004), 182-194.

[47] Z. Yin, Global solutions to a new integrable equation with peakons, Indiana Univ. Math. J. 53 (2004), 1189-1210. 